\title{
Congenital malaria in Europe: review of the literature
}

Lorenza Romani', Hyppolite Tchidjou Kuekou'1, Stefania Bernardi', Emma Manno', Lorenza Putignani ${ }^{2}$, Paolo Palma ${ }^{3}$

1. Bambino Gesu Children's Hospital-IRCCS, Immunological and Infectious Disease Unit, University Department of Pediatrics, Rome, Italy

2. Bambino Gesù Children's Hospital-IRCCS, Unit of Human Microbiome- Unit of Parasitology, Rome, Italy

3. Bambino Gesù Children's Hospital-IRCCS, Head of the Research Unit in Congenital and Perinatal Infections Academic Department of Pediatrics Division of Immune and Infectious Disease, Rome, Italy

\section{Background}

Congenital malaria $(\mathrm{CM})$ is among the less common features of the disease, and neonatal infection remains extremely rare both in endemic and non-endemic areas. P.falciparum is the main responsible of CM in endemic countries in contrast to non-endemic area where P.vivax and P.malariae are reported more frequently. We report a review of the literature about CM in Europe.

\section{Methods}

Case reports and reviews published in the last 30 years were used as sources to identify all the cases of CM in Europe and to summarize clinical features, diagnostic tool and treatment. We use standard search strategy using as key words on PUBMED "congenital malaria", "congenital malaria case reports" and "malaria in non-endemic countries". We excluded all the case reports about CM in endemic countries.

\section{Results}

In the last 30 years, 35 cases of CM were reported in Europe. Among reported cases, 21 (58\%) were caused by P.vivax. Maternal history of malaria may not occur and it is not a criteria for the diagnosis of CM. In most cases of CM, the diagnosis is made at 10-28 days of age and among the reported cases it was diagnosed after 21 days in 15 of 36 cases (42\%). The symptoms are rarely at birth possibly because of the presence of IgG transferred from mother during the pregnancy and protective effect of $\mathrm{HbF}$. The clinical manifestations of $\mathrm{CM}$ do not differ significantly from bacterial or viral sepsis.

\section{Discussion}

Considering the migratory flow from endemic area to non-endemic area, CM should be included in the differential diagnosis of neonatal sepsis in neonates born to women from malaria endemic countries in order to avoid a delayed diagnosis. 